\section{Keine Legalisierung der aktiven Sterbehilfe}

Das niederländische Parlament hat ein Gesetz angenommen, welches die sogenannte aktive Sterbehilfe legalisiert. Unter aktiver Sterbehilfe versteht man die absichtliche Ausübung von Handlungen, welche zur Verkürzung des Lebens eines todkranken Patienten führen sollen. Die aktive Sterbehilfe wird oftmals als Euthanasie bezeichnet. Im Gegensatz dazu steht die passive Sterbehilfe, d.h., die Unterlassung aktiver Massnahmen zur Lebensverlängerung beim Todkranken.

Ich bin gegen eine Legalisierung der aktiven Sterbehilfe in der Schweiz. Für den Arzt bleibt auch bei einem Patienten mit unheilbarer Krankheit die Aufgabe bestehen, körperliche und seelische Leiden zu lindern. Die absichtliche Tötung eines Menschen darf niemals Aufgabe des Arztes werden. Ich anerkenne hingegen, dass es Fälle gibt, in denen aktive Sterbehilfe als Ausdruck tiefen Mitleids geleistet wird. Hier soll die menschliche Gerichtsbarkeit verzeihen. Es besteht jedoch ein grosser Unterschied zwischen Verzeihung einer grundsätzlich verbotenen Handlung, die aus tiefem Mitleid erfolgte, und der ausdrücklichen Legalisierung einer solchen Handlung.

Zweifellos ist es zu einfach und auch heuchlerisch, die aktive Sterbehilfe als Verbrechen zu bezeichnen. Das Gesetz verbietet es, einem Mit- menschen das Leben zu nehmen. Die praktische Medizin indessen wird mit Situationen konfrontiert, in denen die Möglichkeit der aktiven Sterbehilfe sich aufzudrängen scheint. Nicht selten wird der Arzt durch den Sterbenden oder durch dessen Familie gebeten, die Leiden zu verkürzen, wenn alle Therapien versagt haben. Es ist deshalb verständlich, wenn die Niederlande versucht haben, einer stillen Usanz einen gesetzlichen Rahmen zu geben. Die aktive Sterbehilfe darf jedoch niemals zu einer harmlosen Tat oder gar zu einer Routinehandlung werden. Die Tötung eines Menschen muss grundsätzlich ein Tabu bleiben.

Die passive Sterbehilfe hingegen gehört zu den Aufgaben des Arztes. Ärztlich verschriebenes Morphin gestattet, unerträgliche Qualen zu lindern. Ebenso wichtig ist die Liebe zum sterbenden Patienten. Allzulange hat die Medizin der Entwicklung palliativer Pflege zu wenig Aufmerksamkeit geschenkt.

Die Legalisierung der aktiven Sterbehilfe lässt mangelnden Respekt vor dem Leben erkennen. Es ist nur ein kleiner Schritt von der aktiven Sterbehilfe aus Mitleid zur Sterbehilfe aus ökonomischen Gründen, z.B. zur Entlastung der Spitäler von hoffnungslos erkrankten Patienten mit grossem Behandlungsaufwand. Die moderne Gesellschaft ist nicht mehr imstande, ihre Sterbenden zu begleiten. Diese sind in ihren Augen unbequem und unnütz geworden. Die Legalisierung der aktiven Sterbehilfe erscheint in diesem Licht als erschreckende Bequemlichkeit.

PD Dr. Jean Henri Dunant, Nationalrat 